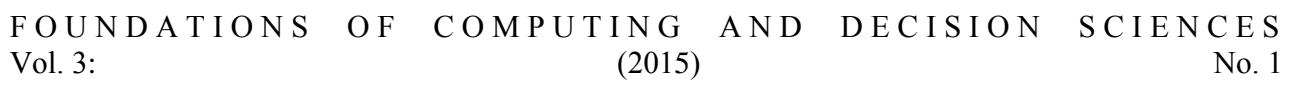

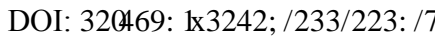

\title{
CLAR: A NOVEL CLUSTER ORIENTED AGENT BASED ROUTING PROTOCOL FOR MANET
}

\author{
Ditipriya SINHA ${ }^{1}$, Uma BHATTACHARYA ${ }^{2}$, Rituparna CHAKI ${ }^{3}$
}

\begin{abstract}
In this paper we propose a novel algorithm CLAR to build an agent based routing protocol using ACO technique in cluster based mobile ad-hoc networks. Clustering makes possible hierarchical routing in which paths are recorded between cluster-heads instead of nodes. This reduces routing overheads. Presence of an unstable and non-reliable cluster-head degrades the performance of the network since cluster-heads of the network take active role in routing messages between a source destination pair. The proposed algorithm chooses the most reliable and stable node as cluster-head depending on four criteria i.e.: stability, battery power, degree, and trust value of the node. It also uses a multiagent based routing algorithm to generate load balanced routes between source and destination in cluster-based network. Performance of CLAR is compared with other existing efficient algorithms such as AODV, CBRP, Wu \& Li's Algorithm, dominating set based clustering algorithms, agent-based algorithms named as SACO and MALBACO. Simulation results prove that the proposed protocol combines merits of both clustering approach and agent based approach and provides efficient packet delivery even in the presence of high load in the network.
\end{abstract}

Keywords: MANET, Dominating Node, Cluster-head, Gateway node, Routing protocol, Ant Colony Optimization

\section{Introduction}

The mobile nature of MANETs leads to frequently changing topology; a route between a

\footnotetext{
${ }^{1}$ Assistant Professor, Department of Information Technology, CIEM, Kolkata 700040,India. Email: ditipriya_sinha@yahoo.co.in Phone:+919836732484

${ }^{2}$ Professor, Department of Computer Science \& Technology, Bengal Engineering \& Science University, Shibpur, Howrah 711103, India. Email: ub@cs.becs.ac.in Phone:+919830899022

${ }^{3}$ Associate Professor, Department of Computer Science \& Engineering,West Bengal University of Technology, Kolkata 700064, India. Email: rchaki@ieee.org Phone: $+919830507213$
} 
source destination pair does not remain valid after a while. Battery life, loss of packets due to mobility, network partition, and transmission error are some of the inherent problems of MANETs. Most of the routing topology used by wired networks fails in case of MANETs. As compared to traditional wired links, the wireless links have significantly lower capacity, that cause the assignment of routing paths more challenging. The ever-increasing level of congestion also creates difficulties in assigning the route in MANET. The wide application coupled with inherent problems makes this field an important area of research. At present research works on cluster based and agent based communication between nodes in a MANET are gaining popularity.

Cluster based routing is aimed to solve the problem of nodes' heterogeneity, and also to limit the amount of routing information propagating inside the network. The idea behind clustering is to group network nodes into a number of disjoint or overlapping clusters. In a cluster, nodes whose priority are higher than other nodes in the network is called dominating nodes. The dominating node that coordinates cluster activities is selected as the cluster-head. Cluster-heads of the clusters take active role in routing messages between a source destination pair. Gateway nodes are used for inter-cluster communication.

Another hot topic of research over routing in MANETs concentrates over agent based ant colony optimization technique. This technique follows the natural behavior of ants for searching foods. Ants deposit a substance called pheromone on the ground as they move in search of food. Other ants can smell this pheromone, and their path-taking decisions are influenced by the pheromone deposition. The pheromone evaporates at an exponential rate, implying that the strength of the pheromone encountered by another ant is a function of the original pheromone strength and the time since the trail was laid.

Clustering makes possible hierarchical routing in which paths are recorded between cluster-heads instead of between nodes. This reduces routing overheads. Presence of an unstable and non-reliable cluster-head degrades the performance of the network since cluster-heads of the network take active role in routing messages between a source destination pair. So selection of a stable and reliable cluster-head is very important in cluster based network. On the other hand agent based approach uses ant colony optimization technique which is used to find shortest route between source-destination pair in ad-hoc networks. But, a few of them are concerned about load and congestion in the network.

In this paper we propose a novel algorithm to build an agent based routing protocol using ant colony optimization techniques in a cluster based mobile ad-hoc network. In this algorithm clustering set up phase is accomplished by using dominating set of nodes. A cluster-head of good quality is defined in this paper by its stability, battery power, connectivity or degree, and trust value. Gateway nodes are also selected from the set of dominating nodes. The proposed algorithm CLAR chooses the most reliable and stable node in a cluster as the cluster-head. It also uses a multi-agent based routing algorithm using ACO to generate load balanced routes between source-destination pairs in a cluster based network.

In this paper section 2 contains review of past works in the area of routing protocols and scope of the work is mentioned in section 3. Section 4 describes proposed work. In section 5 performance of the proposed work has been evaluated. Conclusion has been drawn in section 6 . 


\section{Review of past works}

A categorized review covering the basic routing protocol, cluster based routing protocol and agent based routing protocol is presented in this section.

\subsection{Basic routing protocols}

Destination Sequenced Distance Vector (DSDV) [4, 5] routing protocol is one of the first routing protocols for ad hoc networks. A number of routing protocols have been proposed based on this distance-vector approach, e.g. the Extended Bellman-Ford (EXBF) [6] algorithm. The EXBF protocol is free from routing loops and count-to-infinity problem.

Dynamic Source Routing (DSR) [9] protocol is an on-demand protocol that establishes a route by flooding route request packets in the network. The major limitation of this protocol is that the route maintenance mechanism fails to repair a broken link locally. Ad hoc On Demand Vector (AODV) [7, 8] routing protocol offers loop-free routes even while repairing broken links and is also scalable to a large extent. One of the disadvantages is that the intermediate nodes may have stale entries leading to inconsistent routes.

\subsection{Cluster based routing protocols}

The basic routing protocols show degraded performance as the number of nodes increases. Individual nodes face power shortage due to extra load. The cluster based approach tries to reduce the load on individual nodes, as well as increases scalability and efficiency of routing protocols in mobile ad-hoc networks. The two main tasks involved in maintaining a cluster based network are finding the cluster-head nodes and partitioning the network into clusters. The following section presents review of cluster based routing protocols.

CBRP $[22,18]$ is the example of this type of routing protocols. CBRP divides the nodes in ad-hoc network into a number of disjoint or overlapping clusters. Every node upon receiving "HELLO" message from its neighbour compares its own id with other. If a node distinguishes its own id is the lowest id between its neighbours, this node declares itself as the cluster head. A cluster-head is elected for each cluster to maintain cluster membership information. Inter-cluster routes are discovered dynamically using the cluster membership information kept at each cluster-head. A cluster-head elects a gateway node to communicate with other cluster-heads. CBRP is based on source routing similar to DSR. This CBRP protocol efficiently minimizes the flooding traffic during route discovery and speeds up route discovery process. Too much traffic due to interference is the shortcoming of this protocol.

Minimal energy consumption is one of the goals of cluster based routing protocols. CRAM [16, 30], AWCBRP [25] and SEDEEC [2] are concerned about this. CRAM selects Multi-Cluster-Heads in every cluster since one cluster-head in a cluster may be unreliable and may cause losing energy in case of node failure. This improves energy efficiency and enhances data transmission reliability in MANETs. CRAM however fails to adapt to topology changes. AWCBRP is energy efficient and is based on adaptive weighted cluster based routing protocol which amends swiftly to the topological changes and establishes the 
routing efficiently. This involves high link breakage rate and so partitioning rate of the network is considerably high leading to increase in computational overhead. To increase the life time of whole network SEDEEC is designed. SEDEEC refers to Stochastic and Equitable Distributed Energy Efficient Clustering applied on a heterogeneous network with base station located far away from the network. It introduces two level hierarchical concept, which allows better use and optimization of the energy dissipated in the network.

Wu \& Li's algorithm [29] proposes Connected Dominating Set (CDS) based algorithm for clustering in mobile ad-hoc networks. The main objective of this algorithm is to find the minimal connected dominating sets so that overhead in the network is reduced by decreasing the quantity of cluster-heads. A Dominating Set Based Clustering Algorithm [10] is an extension of $\mathrm{Wu} \&$ Li's method. This algorithm performs well in dense mobile networks, but it is neither scalable, nor resource-balanced. Scenario-based Clustering Algorithm for Mobile ad hoc networks (SCAM) [28] and (k,r)- Dominating set based weighted and adaptive clustering algorithm [26] are two adaptive and distributed clustering algorithms. Quality of a cluster-head is calculated based on different metrics such as connectivity, stability and residual battery power. Stable cluster formation is the main objective of these algorithms. DEMAC [1] is another cluster based routing protocol where cluster head selection is based on residual energy and mobility of the node. SRCHS [13], a Stable Reliable Cluster-head Selection Protocol for mobile ad hoc networks is proposed by the authors. SRCHS selects dominating nodes and non-dominating nodes on the basis of their weight, where weight of a node depends on degree, stability, residual energy and trust value of the node which makes the cluster-head not only stable but also reliable. However actual routing of packet is not considered in it.

Cluster based routing protocol for Delay-Tolerant Mobile Networks (DTMN) [3, 15] uses distributed clustering. Mobility patterns of all nodes are learnt autonomously and on the basis of that clusters are formed. The nodes in a cluster interchangeably share their resources for reducing overheads and balancing resources. This way it increases scalability and efficiency of routing for DTMN.

\subsection{Agent based routing protocols}

Agent based approaches have become popular enough in reducing loads on individual nodes while trying to optimize the performance. This review is based on research in agent based approaches based on ant colony optimization (ACO) for routing and load balancing in an ad-hoc network. There are mainly two different types of agent based routing logic single agent, and multi agent. The following section presents a categorized review of ant colony optimization based routing protocols.

SACO [17] is an agent based routing protocol created to solve the problem of finding shortest path between two nodes in a network. The main computational expense of SACO lies in the updation of pheromone level and calculation of probabilities. It fails to adapt to the dynamic topology. ANT-NET [27], a multi-path routing protocol is a useful technique for dynamic routing in packet switched network. Route discovery is done by using Forward Ants (FANT) and Backward Ants (BANT) as in ANT-NET. Here every ant carries a stack which contains the whole route from a source to a destination. It suffers from bandwidth allocation and hop-count drawbacks and thus it leads to limited scalability. Ant Based Control $(\mathrm{ABC})[14]$ replaces routing tables with pheromone table. It is concerned with 
routing in packet-switched networks and is the first application of ACO to dynamic problems. The main advantage of it is the dynamic property, which results in robustness under various networks and increase of scalability. This technique has a way of avoiding congested nodes. A new proactive routing protocol in MANETs [21] proposes a proactive ant based routing approach for MANETs inspired by the ACO paradigm. This algorithm proactively sets up multiple paths between source destination pairs. It does not follow any features of reactive routing protocols. AntHocNet [24] is a hybrid ACO-routing algorithm. It is a combination of reactive and proactive techniques. The reactive paths are only setup at the start of a session. Proactive paths are set up during maintenance and updated next. It does not give any mechanism for cycle detection. ARA [19] an ACO-based routing scheme using distance vector routing which detects cycle. Route discovery in ARA is done by using Forward Ants (FANT) and Backward Ants (BANT) as in ANT-NET. Once the route is established, packet transmission starts. ARA fails to provide expected throughput with high network load and multimedia data. A parallel ant colony optimization algorithm for all pair routing in MANETs [20] focuses on demand routing using meta-heuristic search technique based on swarm intelligence. This algorithm also develops mechanism to detect cycle. But it also does not take care of load and congestion in the network.

LCRACO [11] is the example of multi agent based load balanced routing of ACO. Connection is established on a multi path basis to balance the load in the network. This algorithm uses two colonies of ants (red and blue) to search for the load balanced path. MALBACO [12], an improved version of LCRACO is another new multi-agent load balancing ant colony optimization algorithm for MANETs. In MALBACO, a node selects the optimized route on the basis of probability of pheromone concentration.. In this protocol probabilities of choosing the same path by two colonies of ants are also reduced. In this way load remains balanced and congestion becomes controlled in MANETs. On the other hand Load balancing of Ant Based Algorithm in MANET [23] proposes an efficient ant based algorithm for data transfer in MANETs.

\section{Scope of the proposed work}

The review leads to the observation that both clustering and ACO techniques have been proved to be quite useful in routing data packets in MANETs. A few limitations still remain.

A successful approach for dealing with maintenance of mobile ad-hoc networks is by partitioning the network into clusters. Clustering is a method that aggregates nodes into group which helps improve routing at network layer by reducing the size of the routing table and in turn the transmission overhead which occurs due to the updation of routing tables after change in topology. Stability and reliability of cluster based approach depend on quality of cluster-heads and gateway nodes in the network. If quality of cluster-head is poor, performance of the network gets also degraded. It is obvious that a cluster-head will be more stable and reliable if we consider its trust value, degree, the residual battery power and mobility as parameter to the election of cluster-head. Selection of gateway nodes also plays an inevitable role in clustered network which needs to be taken care of.

ACO technique is the example of agent based approach. The swarm intelligent mechanisms present in natural ant colonies are used by a powerful class of optimizing 
algorithms. ACO techniques give optimum shortest route between a source destination pair. Very a few ACO based routing protocols such as LCRACO and MALBACO are concerned on balancing load and reducing congestion in the network.

The proposed algorithm 'CLAR' addresses the problems of formation of cluster, selection of stable, reliable, and powerful cluster-head and gateway nodes in cluster based MANETs and then routing packets using multiple agents for balancing load and reducing congestion in the network. Thus proposed protocol combines merits of both cluster based and agent based approaches.

\section{Proposed work}

Proposed work has been described fully in this section.

\subsection{Assumptions}

i) Mobility model used here is random waypoint [31]. This model restricts movement of the mobile nodes to a rectangle. Each node picks a destination within the rectangle along with a speed. The node travels to the destination at that speed. Upon reaching the destination, the node selects and waits for a uniformly distributed pause time. After waiting, the node picks another destination and another speed, continuing the process. The parameters of this model are the minimum and maximum speed and the maximum pause time.

ii) No path loss exists.

iii) Knowledge of the location coordinates is assumed to be known for all nodes by using a GPS like scheme.

iv) All members of a cluster are within $r$ hop distance.

v) Each cluster in the network consists of 4 sectors and each sector has at least one gateway node.

\subsection{Data Dictionary}

- $\quad$ domnode $\mathrm{i}_{\mathrm{i}}$ : It describes $\mathrm{i}^{\text {th }}$ dominating node in a cluster.

- $\quad c h-\mathrm{id}_{\mathrm{i}}$ : It defines the cluster-head of $\mathrm{i}^{\text {th }}$ cluster.

- $\quad$ CH: A variable used to represent a specific cluster-head or a node in the path in between destination-cluster-head and destination.

- $\quad \mathrm{W}_{\mathrm{i}}$ : Weight of node $\mathrm{i}$.

- $\operatorname{cost}_{\mathrm{i}, \mathrm{j}}$ : It is the cost of a link connecting node $\mathrm{i}$ and node $\mathrm{j}$. It is defined as the distance between node $\mathrm{i}$ and $\mathrm{j}$ during specific time interval.

- $\quad$ rmax: Maximum amount of red pheromone amongst all available links at a particular time.

- bmax: Maximum amount of blue pheromone amongst all available links at a particular time.

- $\quad$ forward_count $t_{\mathrm{i}}$ : Number of messages sent by Monitor successfully via node i. 
- $\quad$ tot_forward_message $\mathrm{i}_{\mathrm{i}}$ : Total number of messages sent by the MONITOR via

- node i.

- $\mathrm{G}_{\mathrm{i}}$ : Gateway node of $\mathrm{i}^{\text {th }}$ cluster

- $\quad \mathrm{x}$ : Total number of messages to be sent from a source to a destination.

\subsection{Definitions}

Definition 1: Mobility of a node $i$ having $n$ number of neighbours is defined as the summation of difference of distance computed between the node and all its neighbours during time interval $\Delta \mathrm{t}$, i.e., $\operatorname{mob}_{\mathrm{i}}=\Sigma_{\mathrm{s}=1 . . \mathrm{n}} \operatorname{dist}(\mathrm{i}, \mathrm{s})$ where, $\operatorname{dist}(\mathrm{i}, \mathrm{s})=\left|\mathrm{d}_{\mathrm{si}}^{\mathrm{t}}-\mathrm{d}^{\mathrm{t}+\Delta \mathrm{t}}{ }_{\mathrm{si}}\right|$ where $\mathrm{s}$ is a neighbour of node $\mathrm{i}, \mathrm{d}_{\mathrm{si}}^{\mathrm{t}}$ indicates distance between node $\mathrm{s}$ and node $\mathrm{i}$ at time $\mathrm{t}$. The distance is calculated from the location co-ordinates of the nodes at that instant of time. Stability of the node is defined as the inverse of its mobility.

Definition 2: Cost $_{i, j}$ on a link between node $i$ and $j$ is defined as the distance between node $i$ and node $\mathrm{j}$ during a specific time interval.

Definition 3: Total battery power consumption $b_{p}$ of a node $i$ is proportional to energy required for forwarding and receiving number of packets and also size of packets transmitted and received by the node during a specific time interval.

Definition 4: Probability of concentration of red and blue pheromone between source node $\mathrm{i}$ and destination node $\mathrm{j}$ is defined in the following way:

$$
\begin{gathered}
\operatorname{rprob}_{i j}=\operatorname{red}_{i j} / \text { phsum }_{\text {current }} \\
\text { bprob }_{i j}=\text { blue }_{i j} / \text { phsum }_{\text {current }} \\
\text { phsum }_{\text {current }}=\text { phsum }_{\text {prev }}+\text { red }_{i j}+\text { blue }_{i j}
\end{gathered}
$$

where,

phsum $_{\text {prev }}$ is the total amount of red and blue pheromone deposited between node $\mathrm{i}$ and next hop node $\mathrm{j}$ at time $\mathrm{t}$. Initially phsum $_{\text {prev }}=0$.

phsum $_{\text {current }}$ is the total amount of red and blue pheromone deposited between node $i$ and next hop node $\mathrm{j}$ at time $\mathrm{t}+\Delta \mathrm{t}$.

$\operatorname{red}_{\mathrm{i}, \mathrm{j}}$ : It defines red pheromone value of the link between node $\mathrm{i}$ and next hop node $\mathrm{j}$. It is initialized with summation of trust value and battery power of node $i$.

blue $_{\mathrm{i}, \mathrm{j}}$ : It defines blue pheromone value of the link between node $\mathrm{i}$ and next hop node $\mathrm{j}$. It is initialized with summation of degree and stability of node $i$.

rprob $_{i, j}$ is the probability of deposition of red pheromone between node $i$ and next hop node j.

bprob $_{i, j}$ is the probability of deposition of blue pheromone between node $\mathrm{i}$ and next hop node $\mathrm{j}$. 
These red and blue pheromone probabilities are used for path selection by red and blue ants respectively.

Definition 5: A node i is dominating if its weight crosses threshold limit. Weight of the node $\mathrm{i}$ depends on its trust value $\left(\mathrm{ta}_{\mathrm{i}}\right)$, battery power $\left(\mathrm{bp}_{\mathrm{i}}\right)$, degree $\left(\mathrm{deg}_{\mathrm{i}}\right)$ and stability $\left(1 / \mathrm{mob}_{\mathrm{i}}\right)$. Dominating status will be determined using the following relations and the logic:

Weight of node i i.e

$$
\begin{gathered}
W_{i}=t a_{i}+\operatorname{deg}_{i}+1 / \operatorname{mob}_{i}+b p_{i} \\
\text { select }=(1 / p) \sum_{j=1}^{p} W_{j}
\end{gathered}
$$

where $\mathrm{p}$ is number of the neighbour nodes of node $\mathrm{i}$.

if $\mathrm{W}_{\mathrm{i}}>=$ select, Node $\mathrm{i}$ is declared as the dominating node .

Definition 6: Trust value $\mathrm{ta}_{\mathrm{i}}$ of a node $\mathrm{i}$ is defined as follows:

$$
\text { ta }_{i}=\text { forward _count }_{i} / \text { tot }_{-} \text {forward _message }_{i}
$$

\subsection{Description of proposed protocol}

Proposed protocol is divided into following three sub-sections i.e.: Initialization of cluster formation, Entry of a new node and Routing protocol.

\subsubsection{Initialization of cluster formation}

Initialization of cluster formation is divided into three components. Assign-weight( ) in the following subsection A will assign some weight to all nodes depending on battery power, mobility, degree, and trust value of the node. Initialize-cluster ( ) in the subsection B is used to form a single cluster initially and Initialize-set-of-clusters ( ) in the subsection $\mathrm{C}$ is finally described to form an initial set of clusters.

\section{A. Assignment of weight to each node in the network (Assign-weight( ))}

Step 1: Any node i advertises its presence by broadcasting a NEIGHBOURREQUEST message whose format is \{sequence-number, node-id-i\}.

Step 2: The nodes within one hop distance send an ACK message of format ssequencenumber, node-id-i, first-hop-id $\}$ to the initiator node with in a predefined time period.

Step 3: All such nodes are added to the neighbour list of the node $i$.

Step 4: Node i sends its neighbour list, battery power and degree to one of its neighbour called as Monitor. 
Step 5: Monitor observes mobility of the initiator node for some time interval $t_{\text {mon }}$ and then calculates its mobility by using Definition 1 [Section 4.3].

Step 6: During this period $t_{\text {mon }}$ Monitor sends some messages to another node present in the neighbour list using node $\mathrm{i}$ as the intermediate node.

Step 7: Depending on the number of successful transmission of a number of messages, Monitor assigns a trust value to the initiator node using Equation 4.6 in Definition 6 [Section 4.3]. Monitor calculates weight of the node i using Equation 4.4 in Definition 5 [Section 4.3]. If weight of the node is greater than select [Definition 5, Section 4.3], the status of the node is a dominating one by Definition 5 [Section 4.3].

Step 8: Monitor sends weight and status of the node $i$ to the node $i$. Thus, the node $i$ is aware of its weight and status.

Step 9: The process from step 1 to step 8 will be repeated for every node present there.

Step 10: Stop.

\section{B. Initialization of cluster formation (Initialize-cluster( ))}

Step 1: Any node called as initiator broadcasts FINDNEIGHBOUR message to its neighbours. Message format is \{sequence-number, initiator-node-id, hop-count $\}$. Hop-count is initially 0 . Value of the final hop-count is considered as $r$.

Step 2: When it reaches to next-hop nodes, they send the ACK message to the initiator. The ACK message contains the information \{sequence-number, initiator-node-id, next- hopneighbour-id $\mathrm{d}_{1}$, status, $\mathrm{W}_{\mathrm{id} 1}$, hop-count $\}$, where $\mathrm{W}_{\text {id } 1}$ is the weight of the first-hopneighbour node -id1 and status indicates whether the node is a dominating one or not. Hopcount in ACK message is increased by the value 1.

Step 3: The nodes which send the ACK message to the initiator, again broadcast FINDNEIGHBOUR message to other nodes in the next hop which contains the information as follows : \{sequence-number, initiator-node-id, next-hop-neighbour-id ${ }_{1}$, hop-count\} .

Step 4: The ACK message which reaches the initiator node from 2 hop distance (i.e. hopcount=2) within a specific time interval contains the message format \{ sequence-number, initiator-node-id, next-hop-neighbour-id ${ }_{1}$, next-hop-neighbour-id ${ }_{2}$, status, $\mathrm{W}_{\mathrm{id} 2}$, hop-count $\}$, where $\mathrm{W}_{\mathrm{id} 2}$ is the weight of the neighbour $\mathrm{id}_{2}$ and status indicates whether the node nexthop-neighbour $\mathrm{id}_{2}$ is a dominating one or not.

Step 5: Hop-count is increased by 1 and broadcasting FINDNEIGHBOUR message and sending ACK message by next hop nodes will be repeated so long as hop-count becomes equal to $r$. Final ACK message from $r$ hop distance which reaches the initiator node contains the following information:\{sequence-number, initiator-node-id, next-hopneighbour-id $\mathrm{i}_{1}$, next-hop-neighbour-id $\mathrm{i}_{2}, \ldots .$. , next-hop-neighbour-id $\mathrm{d}_{\mathrm{r}}, \mathrm{W}_{\mathrm{idr}}$, status, $\mathrm{W}_{\mathrm{idr}}$, hopcount \}, where $\mathrm{W}_{\text {idr }}$ is the weight of the next-hop-neighbour-id $\mathrm{r}_{\mathrm{r}}$ at $\mathrm{r}$-hop distance from the initiator-node and status indicates whether the next-hop-neighbour-id $\mathrm{r}_{\mathrm{r}}$ is a dominating one or not.

Step 6: Cluster is formed by those neighbours at a distance $<=$ r from which ACK message reaches the initiator node within a predefined time interval. 
Step 7: If any node gets more than one FINDNEIGHBOUR message from many initiators simultaneously, the node will send ACK message to any one of them and the other FINDNEIGHBOUR message will be discarded by it.

Step 8: Dominating node having highest weight among them is selected by the initiator node as a cluster-head $(\mathrm{CH})$ and the initiator node transfers its neighbour list to the cluster head $\mathrm{CH}$.

Step 9: $\mathrm{CH}$ advertises its identity to all nodes in its neighbour-list.

Step 10: Cluster-head $\mathrm{CH}$ divides the cluster into 4 sectors. In each sector, node having highest weight is selected as a gateway node.

Step 11: Stop

\section{Set of cluster formation (Initialize-set-of-clusters( ))}

Step 1: Some nodes which are separated among themselves by more than $2 \mathrm{r}$ hop distance are selected randomly. Procedure Initialize-cluster ( ) in subsection 4.4.1.B is executed for each of the selected nodes which leads to form a set of clusters with their members, stable cluster-heads and gateway nodes in the network for a specified time duration.

Step 2: After a specified time duration, the whole process of Assign-weight( ), and step1 of Initialize-set-of-clusters () is executed to form the new set of clusters in MANETs and this process will go on continuously.

\subsubsection{New node entry within a cluster (New-entry( ))}

Step 1: Step 1-Step 7 of Assign-weight ( ) will be followed considering new node as node i in Assign-weight ( ).

Step 2: Monitor of the new node transfers weight and status of the new node to the clusterhead and also to the new node.

Step 3: If weight of the new node is greater than that of the present cluster-head, the new node advertises itself as new cluster-head and requests the present one to send all neighbour list and gateway members' list to it. Old Cluster-head sends them to the new Cluster-head.

Step 4: Stop

Entry of a new node into a cluster is shown using Figure1, Figure 2 and Figure 3.

\subsubsection{Routing protocol}

Routing protocol will be governed by the algorithm Route-Cluster( ). Route-Cluster( ) during its execution may call another module gateway-route( ) and ACO-Route( ) which are described later. 


\section{A. Module Route-Cluster ( )}

Step 1: Each node in network maintains two pheromone tables-Red and Blue. Each entry of red pheromone table is initialized with summation of trust value and battery power of the node. On the other hand each entry of blue pheromone table is initialized with summation of stability and degree of the node [Definition 4 in section 4.3].

Step 2: For a particular source-destination (S-D) pair, source informs the destination address $\mathrm{D}$ to its respective cluster-head (say ch-id $\mathrm{i}_{\mathrm{i}}$ )

Step 3: If the destination does not belong to this cluster-head ch-id ${ }_{\mathrm{i}}$, ch-id $\mathrm{i}_{\mathrm{i}}$ calls gatewayroute( ) which finds the path from source-cluster-head ch-id ${ }_{i}$ to the destination clusterhead $c h-\mathrm{id}_{\mathrm{m}}$ via intermediate gateway nodes and cluster-heads, $\mathrm{CH}=$ ch-id $\mathrm{m}_{\mathrm{m}}$; Else $\mathrm{CH}=$ ch- $\mathrm{id}_{\mathrm{i}}$

Step 4: Cluster-head CH calls ACO-Route ()

Step 5: Stop

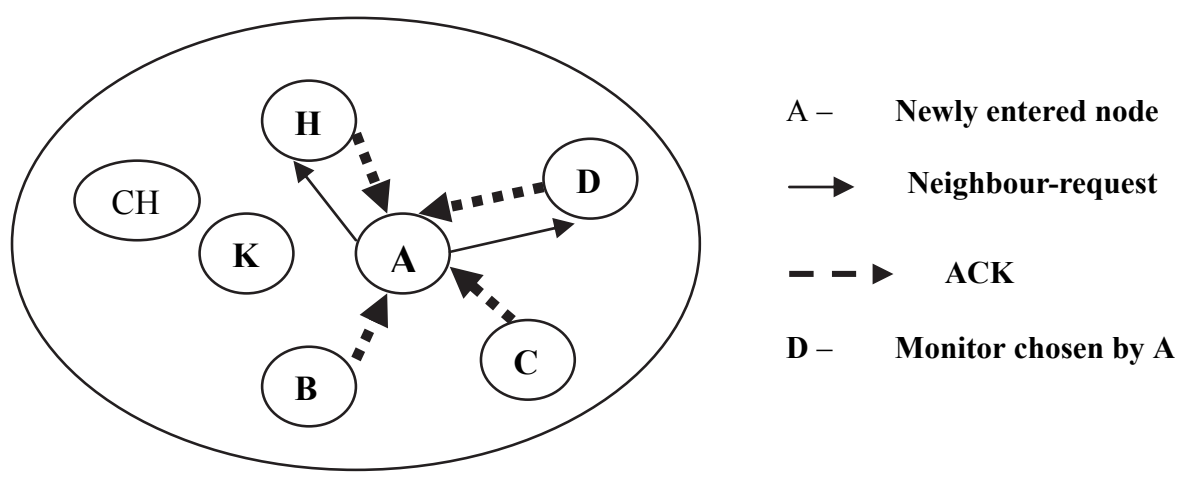

Fig. 1: Monitor selection
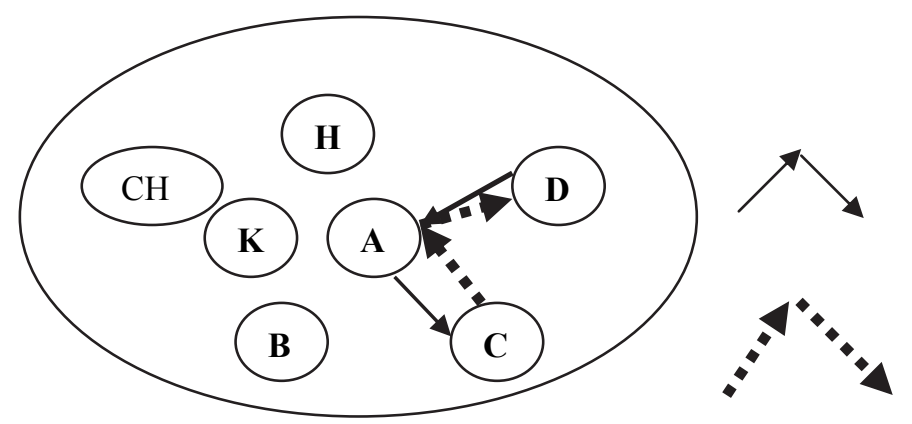

Messages are sent by node $D$ to $\mathrm{C}$ through A to compute trust value of new node $A$

ACK Messages are sent by node $C$ to $D$ through $A$

Fig. 2: Trust value Assignment of new node A by Monitor D 


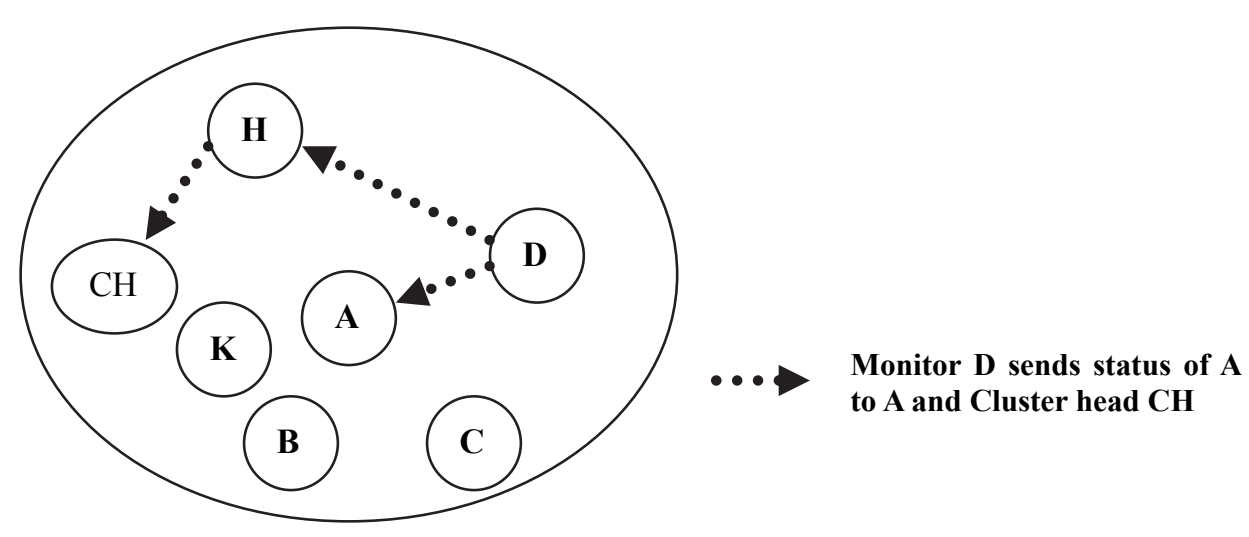

Fig. 3: Monitor D sends status of A to cluster head and node A

\section{B. Module Gateway-route ( )}

Step 1: Cluster-head $\mathrm{Ch}-\mathrm{id}_{\mathrm{i}}$ of cluster $\mathrm{i}$ communicates the destination address to the respective gateway node(s).

Step 2: Gateway node $G_{i}$ of $i^{\text {th }}$ cluster sends ROUTEREQUEST packet to the gateway node $\mathrm{G}_{\mathrm{k}}$ of adjacent (say, $\mathrm{k}^{\mathrm{th}}$ ) cluster. $\mathrm{G}_{\mathrm{k}}$ communicates the destination address to its clusterhead ch-id .

Step 3: If destination is not present in its cluster, step 1- step 2 will be executed again using the cluster-head $\mathrm{Ch}-\mathrm{id}_{\mathrm{k}}$ as the communicating cluster-head and this process will go on till destination-cluster-head is reached.

Step 4: Destination-cluster-head returns ACK message to ch-id ${ }_{\mathrm{i}}$ backtracking in the same route traversed before.

Step 5: Stop

\section{Module ACO-Route ( )}

Step 1: q is initialized with $0 . / * \mathrm{q}$ stands for number of messages to be sent */

Step 2: $\mathrm{CH}$ initializes variable $\mathrm{v}$ with 0 and 1 (in toggling mode) for alternate messages respectively.

Step 3: If $(\mathrm{v}=0) /(\mathrm{v}=1) \mathrm{CH}$ generates $\mathrm{red} /(\mathrm{blue})$ ant and calculates probability of concentration of red/(blue) pheromone $\operatorname{rprob}_{\mathrm{CH}, \mathrm{j}} /\left(\right.$ bprob $\left._{\mathrm{CH}, \mathrm{j}}\right)$ on each available link between $\mathrm{CH}$ and next hop node j using Definition 4 [Section 4.3]. Red/(blue) ant selects link where $\operatorname{rprob}_{\mathrm{CH}, \mathrm{j}} /$ (bprob $_{\mathrm{CH}, \mathrm{j}}$ ) is maximum amongst all available links and sends $\mathrm{q}^{\text {th }} /(\mathrm{q}+1)^{\text {th }}$ message with this link. On any link emanating from $\mathrm{CH}$ to next-hop node $\mathrm{j}$ if (red $\mathrm{CH}_{\mathrm{j}}+$ blue $_{\mathrm{CH}, \mathrm{j}}>=(\max +$ bmax $)$, that link is considered as blocked temporarily. $/ *$ It takes care of reducing congestion in a MANET. */

Step 4: When red/(blue) ant selects $\mathrm{k}$ as the next hop node it deposits red(blue) pheromone between link $\mathrm{CH}$ and $\mathrm{k}$. Rate of deposition of pheromone amount is inversely proportional to the cost of the link cost ${ }_{\mathrm{CH}, \mathrm{k}}$ which helps ants choose lowest cost path. It is calculated as follows: 


$$
\begin{aligned}
& \operatorname{red}_{C H, k}=\operatorname{red}_{C H, k}+\partial / \cos t_{C H, k} \\
& \text { blue }_{C H, k}=\text { blue }_{C H, k}+\partial / \cos t_{C H, k}
\end{aligned}
$$

where $\partial$ is a constant factor. Best value of $\partial$ is determined by using trial and error method.

Step 5: $\mathrm{q}$ is incremented. If $\mathrm{q}<\mathrm{x}$ step $4 \& 5$ are repeated until all messages are sent to nexthop node $\mathrm{k}$ from $\mathrm{CH}$.

Step 6: Battery power consumed by $\mathrm{CH}$ is computed using Definition 3 [Section 4.3] which will be used later for selecting cluster-head in next session.

Step7: During execution of step 4-6, step 7 is executed in parallel. After each specific interval, red/(blue) pheromone is evaporated from all available links $(\mathrm{j})$ emanating from $\mathrm{CH}$ whose amount is proportional to the $\operatorname{cost}_{\mathrm{CH}, \mathrm{j}}$ using following equations:

$$
\begin{gathered}
\operatorname{red}_{C H, j}=\operatorname{red}_{C H, j}+\phi / \cos t_{C H, j} \\
\text { blue }_{C H, j}=\text { blue }_{C H, j}+\phi / \cos t_{C H, j}
\end{gathered}
$$

where $\phi$ is a constant factor. Best value of $\phi$ is determined by using trial and error method.

Step 8: $\mathrm{CH}$ is assigned the value of $\mathrm{k}$. Step 3 to step 7 will be executed by next hop node $\mathrm{k}$ for each message and battery power consumed by next hop node $\mathrm{k}$ is computed by using Definition 3 [Section 4.3]. After each $\Delta \mathrm{t}$ time interval red/(blue) pheromone is evaporated from all available links(j) whose amount is proportional to the cost $k, j$ using similar equations mentioned in step 7.

Step 9: Step 2 to step 8 are repeated until all messages reach the destination node D.

Step 10: Stop

\subsubsection{Algorithm CLAR}

Begin

Execute Assign-weight( )

Execute Initialize-set-of-clusters( ) to initialize the clusters

If any new-node enters in the network, execute new-entry( )

For source destination pair (S, D) execute Route-Cluster( )

End

\section{Performance evaluation}

Performance of proposed protocol is analyzed using simulation techniques. The various parameters considered for performance evaluation are described in section 5.1 and simulation results are described in section 5.2. 


\subsection{Performance metrics}

Performance metrics used to evaluate our proposed protocol are overhead, delivery rate, load, quantity of cluster-heads and number of dominating nodes. Performance of CLAR is compared with three other categories of existing efficient algorithms such as AODV [8], a basic routing protocol CBRP [17], Wu \&Li's algorithm [29] and Dominating Set Based Clustering Algorithm [11], three cluster based algorithms and MALBACO [13] and SACO [18], two agent based algorithms. According to basic characteristics of the above stated routing protocols, proposed protocol is compared with others. For this reason, results and comparison of proposed routing protocol with other existing routing protocols are divided into six subsections in section 5.2.2.

\subsection{Simulation environment and results}

Details regarding simulation are discussed in the following subsections.

\subsubsection{Simulation setting}

This protocol is simulated in NS2.29. Simulation environment of this protocol is Fedora 9. We use IEEE 802.11 for wireless LAN as MAC layer. The channel capacity of mobile node is $2 \mathrm{Mbps}$. In our simulation mobile nodes move in a $600 * 600 \mathrm{~m}^{2}$ region for 125 second simulation time. Mobility model is considered here as random waypoint. It is assumed that each node moves independently with the same average speed $10 \mathrm{~m} / \mathrm{s}$ and pause time is $0-25$ second. No path loss is considered. The network size is varied as 10, 20, 30, 40 and 50 nodes. The simulated traffic is constant bit rate (CBR). Table 1 shows the simulation setting of the network environment.

Table 1. Simulation setting

\begin{tabular}{|c|c|}
\hline Name & Value \\
\hline Channel & Wireless \\
\hline Propagation & Two Way \\
\hline Network Interface Type & Wireless \\
\hline Antenna & Omni Antenna \\
\hline No of nodes & 10 to 50 \\
\hline MAC & IEEE 802.11 \\
\hline Simulation Area & $600 * 600 \mathrm{~m}^{2}$ \\
\hline Timeout Period & $3.0 \mathrm{sec}$ \\
\hline Cluster Contention Interval & $5.0 \mathrm{sec}$ \\
\hline Maximum no. of hops in a cluster $(\mathrm{r})$ & 2 \\
\hline
\end{tabular}




\subsubsection{Results and comparisons}

\section{A. Number of dominating nodes vs. number of nodes in CLAR}

Dominating nodes in the network play inevitable role in selecting cluster-heads in the network. When a large number of nodes in the network become dominating nodes, lots of hazards are created in selecting cluster-heads and overhead in the network also increases.

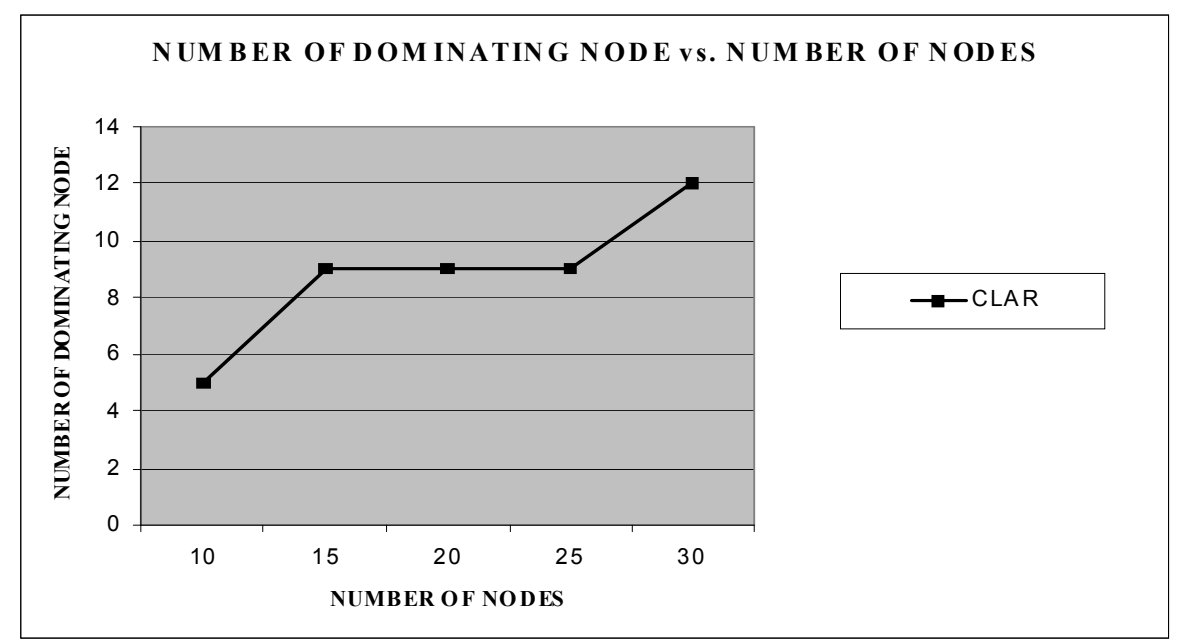

Fig. 4: Number of dominating nodes vs. number of nodes

The graph in Figure 4 shows that as number of nodes increases, number of dominating nodes does not increase rapidly and it remains same and stable when the numbers of nodes vary from 15 to 28 . So, number of clusters also does not increase rapidly which leads to less overhead in the system.

\section{B. Control packet overhead vs. number of nodes}

This sub-section compares CLAR with CBRP; a well known cluster based routing protocol. In CBRP protocol control packets named as LIVE, CTGATEWAY, ARGATEWAY are used whereas CLAR requires to use three control packets, namely FINDNEIGHBOUR, ACK, NEIGHBOUR_REQUEST for cluster formation. On the other hand, routing the protocol CBRP uses the control packets namely RDemand, RResponse, RERR, SEQNUM whereas CLAR uses only ROUTEREQUEST packet.

Thus control packet overhead of CBRP is higher compared to CLAR. Figure 5 shows that performance of CLAR is better compared to CBRP due to its lesser number of control packets. 


\section{Delivery rate vs. number of nodes}

This section compares proposed protocol CLAR with AODV and CBRP. AODV and CBRP are known for their routing efficiency in terms of delivery rate. Figure 6 shows this comparison of the proposed protocol with AODV and CBRP.

AODV is not cluster based routing protocol. In case of cluster-based routing protocols CBRP and CLAR, some time is required for cluster formation and cluster-head selection. Performance of AODV is best in comparison to CBRP and CLAR when number of nodes is small like 20. But when number of nodes in the network is more than 20, delivery rate of CLAR is always better than AODV, since clustering increases efficiency and scalability of routing protocol in mobile ad-hoc networks.

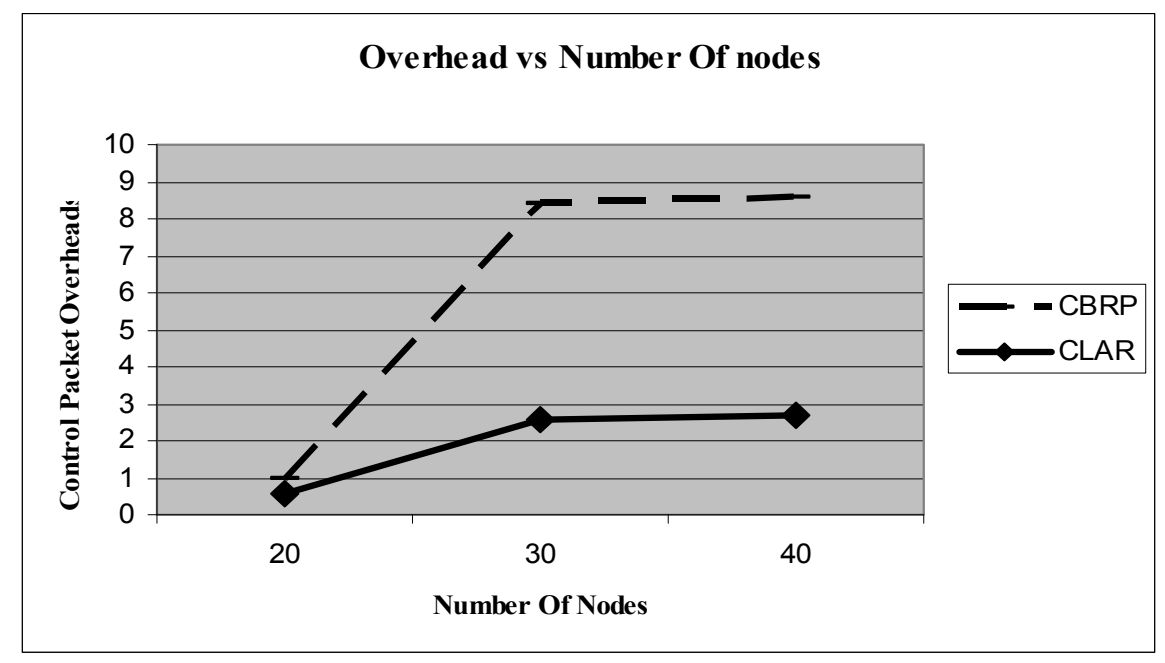

Fig. 5: Control packet overhead vs. number of nodes

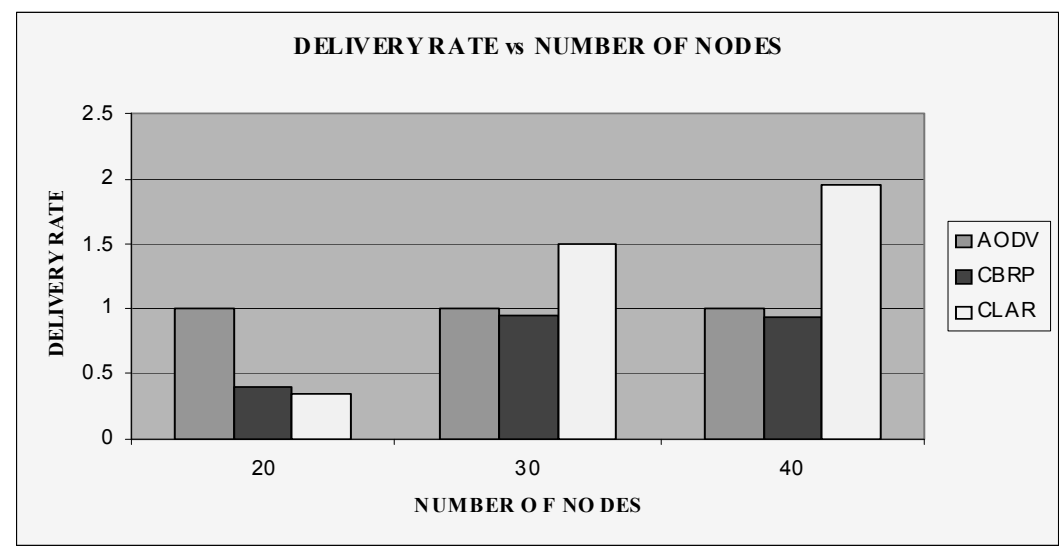

Fig. 6: Delivery rate vs. number of nodes 
On the other hand performance of CLAR is always better than CBRP since CLAR unlike CBRP is based on multi-agent load balanced and congestion controlled routing algorithm.

\section{Quantity of cluster-heads vs. number of nodes}

This section compares proposed protocol with Wu \& Li's algorithm and Dominating Set Based Clustering Algorithm. These two protocols are known for their novelty in clusterhead selections. Main objective of these protocols is to reduce overheads in the network by decreasing the quantity of cluster-heads. Comparison of the proposed algorithm with these algorithms is shown in Figure 7.

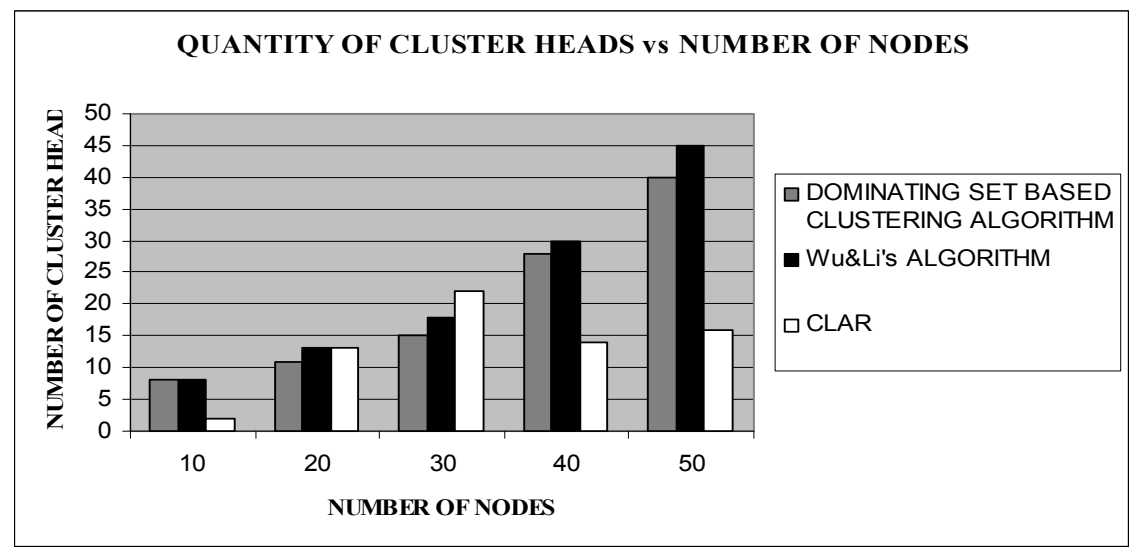

Fig. 7: Quantity of cluster-heads vs. number of nodes

Here, it is found that when number of nodes equals 10 and 20, performance of CLAR is the best amongst three. But when number of nodes equals 30, dominating set based algorithm shows best performance whereas the performance of other two are slightly degraded. When number of nodes in the network is more than 30 performance of proposed protocol CLAR is the best amongst three. The overall performance of CLAR is far better than $\mathrm{Wu} \& \mathrm{Li}$ 's algorithm and Dominating set based clustering algorithm for MANETs.

$\mathrm{Wu} \&$ Li's algorithm and Dominating set based clustering algorithm are based on finding minimal connected dominating sets for selection of cluster-head. In this proposed algorithm CLAR, selection of dominating nodes are made on the basis of four parameters such as its trust value, battery power, degree and stability which make selection of reliable and stable cluster-heads. For this reason in this proposed protocol number of cluster-heads is less compared to other two routing protocols.

\section{E. Delivery rate vs. load}

Both SACO and MALBACO are used for balancing load in the MANET. Proposed protocol CLAR is compared to both of these algorithms and the result is shown in Figure 8. 
Proposed protocol CLAR is a cluster oriented multi agent based routing protocol which balances load and controls congestion in the network. MALBACO is the multi agent based routing protocol only. When load in the system varies from 2 to 6 MALBACO is better than CLAR, but as the load increases CLAR shows better performance. This arises because clustering increases routing efficiency and scalability in the network. SACO is a single agent based routing protocol. For the same reason described above behavior of proposed protocol remains same as before. So, overall performance of proposed protocol is better than both SACO and MALBACO when load increases in the network.

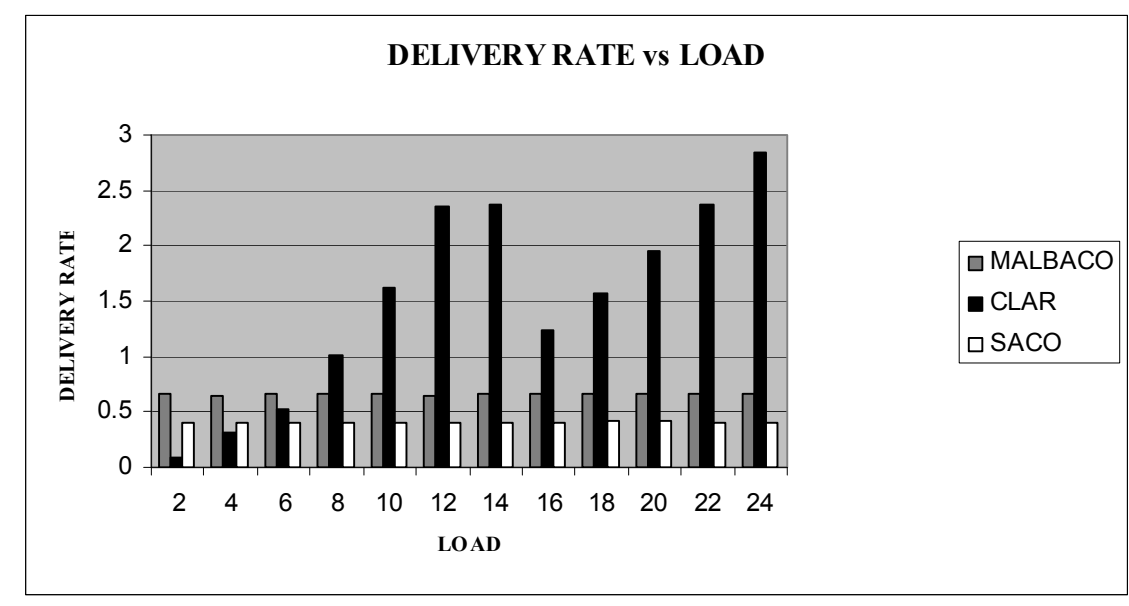

Fig. 8: Delivery rate vs. load

\section{Conclusion}

Proposed protocol combines clustering approach with agent based approach. This protocol selects stable and reliable cluster-heads and gateway nodes. Cluster formation, stable and reliable cluster-head selection, gateway node selection are main objectives of this clustering approach. On the other hand routing in this protocol is implemented applying agent based ACO technique. Here two different colored ants are used for balancing load and reducing congestion in the network. Thus this protocol takes the advantages of both cluster based approach and agent based ant colony optimization technique and applies it successfully in MANET.

It has been observed that number of dominating nodes in CLAR does not increase rapidly with increase in number of nodes in MANET. This result ensures that number of clusters also does not increase rapidly with increase in number of nodes and in turn overhead remains low in the network. In terms of control packet overhead, CLAR has shown better result than CBRP, another cluster based routing protocol.

AODV and CBRP are known for their routing efficiency in terms of delivery rate. From the results discussed in section 5, it is clear that performance of CLAR is better than CBRP 
and AODV in terms of delivery rate. Wu \& Li's algorithm and Dominating Set Based Clustering Algorithm are known for their efficient selection of cluster-head. Main objective of these protocols is to reduce overheads in the network by keeping the quantity of clusterheads low. In this proposed algorithm CLAR, selection of dominating nodes are made on the basis of four parameters such as its trust value, battery power, degree and stability which lead to selection of reliable, stable and powerful cluster-head and gateway nodes in a cluster. It has been noted from our simulation results that number of cluster-heads in CLAR protocol is less than these two protocols mentioned above. SACO and MALBACO are used for balancing load in the MANET. Overall performance of CLAR is better than both SACO and MALBACO when load increases in the network.

At last but not least simulation results prove that the proposed protocol combines merits of both clustering approach and agent based approach. The proposed algorithm chooses most reliable and stable node as cluster head depending on four criteria i.e.: stability, battery power, degree, and trust value of the node. This proves to be beneficial for the stability and the reliability of clusters in a dynamic network. Multiple agents are deployed using the concept of ant colony optimization for more efficient packet routing and balancing load throughout the network. Simulation results of proposed protocol ensure efficient packet delivery even in presence of high load in the network.

Acknowledgement: We do acknowledge the invaluable suggestions made by the reviewers in improving the quality of the paper.

\section{References}

[1] A. Majumder, N. Sarma, DEMAC: A Cluster-Based Topology Control for Ad Hoc Networks, IJCSI International Journal of Computer Science Issues, Vol. 7(5), 2010.

[2] B. Elbihiri, R. Saadane, D. Aboutajdine, Stochastic and Equitable Distributed Energy Efficient Clustering, Int. J. Adhoc and Ubiquitous Computing, Vol. 7(1), 2011.

[3] C. Liu, J. Wu, Scalable Routing In Delay Tolerant Network in Proc. ACM MObiHOC, 2007.

[4] C. Deverapalli, L. Joiner, S-M. Yoo, H. Choo; Performance Comparison of Various Routing Protocols in Mobile Ad Hoc Networks; International Conference on Wireless Networks; LasVegas, Nevada, USA, 2002.

[5] C. E. Parkins, P. Bhagwat; Highly Dynamic Destination Sequence Distance Vector Routing (DSDV) for Mobile Computers, Proceedings of ACM SIGCOMM,1994.

[6] C. Cheng, R. Reley, S.P.R. Kumar, J.J. Garcia-Luna-Aceves; A loop-free extended Bellman-Ford routing protocol without bouncing effect; ACM Computer Communications Review, 1989.

[7] C. E.Perkins, Ad-hoc on-demand distance vector routing, MILCOM '97 panel on Ad Hoc Networks, 1997.

[8] C. E. Perkins, E. M. Belding-Royer, and S. R. Das, Ad hoc On-Demand Distance Vector $(A O D V)$ Routing, IETF Internet Draft, 2003. 
[9] D.B. Johnson, D.A. Maltz; Dynamic Source Routing in Ad Hoc Wireless Networks, Mobile Computing, edited by Tomasz Imielinski and Hank Korth, 1997, pp. 153-181.

[10]D. Cokuslu, K. Erciyes A Dominating Set Based Clustering,Algorithm for MobileAd HocNetworks, MASCOTS '07 Proceedings of the $15^{\text {th }}$ International Symposium on Modeling, Analysis, and Simulation of Computer and Telecommunication Systems, 2007.

[11] D. Sinha, R. Chaki, LCRACO-A New Load and Congestion Control Routing Based On Ant Colony Optimisation, $11^{\text {th }}$ IEEE International Conference on Computer and Information Technology (ICCIT 2008 ), 2008.

[12] D. Sinha, R. Chaki, MALBACO - A New Multi-Agent Load Balanced Ant Colony Optimization Algorithm for MANETs, $8^{\text {th }}$ International Conference On Computer Information System and Industrial Management Application, 2009.

[13] D. Sinha, R. Chaki, SRCHS - A Stable Reliable Cluster-head Selection Protocol, accepted in WiMo-2011, Springer Communications in Computer and Information Science (CCIS) Series, Issue June, 2011.

[14] F. Ducatelle, G. De Caro, L.M. Gamardella, Ant Agents For Hybrid Multipath Routing in MANETs, IDSIA, Galleria2, CH-6928 Manno-Lugano, Switzerland, 2005.

[15] H. Dang, H. Wu, Clustering and Cluster based routing protocol for Delay- Tolerant Mobile Networks, IEEE Transactions On Wireless Communications, Vol. 9, June 2010.

[16] M. Gerla and J.Tasai, Multicluster, mobile ,multimedia radio network, ACM-Baltzer Journal of Wireless Networks, 1997.

[17] M Dorrio,G Di Caro, Mobile Agents For Adaptive Routing, $31^{\text {st }}$ International Conference Hawaii, 1998.

[18] M. Jiang, J. Li, Y.C. Tay, Cluster Based Routing Protocol(CBRP), Functional Specification Internet Draft, draft-ieft-manet-cbrp.txt, 1999.

[19] M. Gunes, U. Sorges, I. Bouazizi, ARA- the ant colony based routing algorithm for MANET, Workshop on Ad-hoc Networks, ICPP Proc of the 2002.

[20] M. Towhidul, P. Thulasiraman, R.K. Thuladiram, A parallel ant colony optimization algorithm for all pair routing in MANETs, IEEE 2010.

[21] M.H. Mamoun A new proactive routing algorithm for MANET, International Journal of Academic Research, 2010.

[22] P. Krishna, N. H. Vaidya, M. Chatterjee, D.K. Pradhan, Cluster Based Routing Protocol, ACM SIGCOMM Computer Communication Review, 1997.

[23] R. Kaur, R.S. Dhillon, H.S. Sohal, A.S. Gill, Load balancing of Ant Based Algorithm in MANET, IJCST, 2010.

[24] S. Rajagopalam, C. Shen, A Routing Suite for Mobile Ad hoc Networks using Swarm Intelligence, Department of Computer and Information Sciences, University of Delaware, Newark, 2003.

[25] S. Karunakaran, P. Thangara An adaptive weighted cluster based routing (AWCBRP) protocol for mobile ad-hoc networks, Journal WSEAS Transactions On Communication, Vol. 7, 2008. 
[26] S.Anitha, M.P, Sebastian ( $k, r)$-Dominating set based weighted and adaptive clustering algorithm, in Institute Of Engineering And Technology, Vol. 5, 2011.

[27] V. Laxmi, L. Jain, M.S. Gaur, Ant Colony Optimization based Routing on NS-2, Proceedings of International Conference On Wireless Communication and Sensor Networks, WCSN 2006.

[28] V.S. Anitha, M.P, Sebastian SCAM: scenario-based clustering algorithm for mobile ad hoc networks, in IEEE Press Piscataway, NJ, USA, Source : Proceedings of the First International Conference on Communication Systems and Networks, Bangalore, India, 2009, pp. 375-382, ISBN:978-1-4244-2912

[29] J. Wu, H. Li, A Dominating Set Based Routing Scheme in Ad-hoc Wireless Sensor Networks, Springer Science Business Media B. V., 2001.

[30] Z. Zheng, Z.Wu, H. Lin and K. Zheng, CRAM: An Energy Efficient Routing Algorithm for Wireless Sensor Networks, Computer and Information Sciences, 2004.

[31] J. Broch, D.A. Maltz, D.B. Johnson, Y. Hu, and J. Jetcheva, A performance comparison of multi-hop wireless ad hoc network routing protocols, Journal of Mobile Computing and Networking, 1998, pp. 85-97.

Received April, 2012 